\section{Spectral-domain optical coherence tomography analysis of persistent subretinal fluid after scleral buckling surgery for macula-off retinal detachment}

${ }^{1}$ Department of Ophthalmology, Sapienza University, Umberto I Hospital, Rome, Italy

${ }^{2}$ Department of Ophthalmology, Polytechnic University of Marche, Ancona, Italy

Correspondence: M Gharbiya, Department of Ophthalmology, Sapienza University, Umberto I University Hospital, Viale del Policlinico 155, Rome 00161, Italy

Tel: +3906 49975302;

Fax: +3906 49975304 .

E-mail: magda.gharbiya@ tiscali.it

Received: 18 August 2014 Accepted in revised form: 14 May 2015

Published online:

3 July 2015

\begin{abstract}
Purpose To determine the predictive value of markers for persistent subretinal fluid (SRF) absorption and the influence of subfoveal fluid on visual outcome after scleral buckle (SB) surgery for rhegmatogenous retinal detachment (RRD).

Patients and methods This was a retrospective, observational study. We reviewed the medical records of 64 eyes of 64 patients who underwent SB surgery for macula-off RRD. Patients underwent clinical examination and spectral-domain optical coherence tomography before surgery, at 1 month and every 3 months postoperatively. The height and width of SRF bleb(s) were measured over time.

Results Persistent SRF at 1 month was observed in 40 eyes $(62.5 \%)$. SRF blebs were first detected $1.7 \pm 2.2$ months postoperatively. In 29 cases that could be fully followed up, SRF blebs were completely absorbed $7.8 \pm 4.4$ months postoperatively. Resolution of fluid was associated with an improvement of VA $(P=0.003)$. Serial measurements of SRF bleb size showed that bleb width decreased significantly at all time points during the 12-month follow-up period $(P<0.05)$, while significant bleb height decrease occurred from postoperative sixth month only $(P<0.05)$. There was no correlation between VA outcomes and subfoveal bleb height or width $(P>0.05)$. The cut-off value of the bleb width-to-height ratio level for predicting bleb absorption at 6 months was 7 , with $89 \%$ sensitivity and $83 \%$ specificity.
\end{abstract}

M Gharbiya ${ }^{1}$, R Malagola ${ }^{1}$, C Mariotti ${ }^{2}$, F Parisi ${ }^{1}$, U De Vico ${ }^{1}$, C Ganino ${ }^{1}$ and F Grandinetti ${ }^{1}$
Conclusions Visual improvement may occur with late resolution of residual subfoveal fluid. A bleb width-to-height ratio $>7$ indicates a higher risk of SRF to persist beyond 6 months after surgery. Eye (2015) 29, 1186-1193; doi:10.1038/eye.2015.113; published online 3 July 2015

Introduction

After surgery for rhegmatogenous retinal detachment (RRD), the finding of persistent subretinal fluid (SRF) has been reported even when the retina appears fully attached on ophthalmoscopy and all retinal breaks are adequately sealed. This entity was probably first described by Machemer after resolution of experimental retinal detachment in owl monkeys. $^{1}$

The advent of optical coherence tomography (OCT) resulted in a number of reports of persistent SRF after RRD surgery. ${ }^{2-13}$ Two patterns of residual SRF have been identified, confluent and bleb-like pockets of fluid and it has been reported that fluid can take more than 12 months to resolve. The presence of persistent SRF has been attributed to several possible causes without, however, conclusive evidence for either vitrectomy vs buckling surgery, the use of encircling vs segmental scleral explant, the use or omission of gas injection, completeness of SRF drainage, or cryotherapy. ${ }^{2-13}$ The incidence of persistent SRF varied widely among the published series, ranging from 9 to $94 \%$. Furthermore, it remains unclear whether the visual outcome is affected: some authors have suggested that persistent submacular fluid does 
not influence visual outcome, $, 8,8,10$ while others have disagreed. $3,4,13$ These conflicting results may have been caused by short follow-up periods, small case numbers, or a lack of quantification of SRF.

In the current retrospective, observational study, patients with successfully repaired RRD after scleral buckle (SB) surgery underwent preoperative and postoperative spectral-domain OCT (SDOCT) to determine the predictive value of markers for persistent SRF absorption as well as the influence of subfoveal fluid on visual outcome. The incidence and the prognostic factors of the persistence of SRF after surgery were also evaluated.

\section{Patients and methods}

We reviewed the medical records of patients with primary RRD involving the fovea (macula-off), who were treated with SB surgery between January 2012 and January 2013. The study protocol was approved by the local ethics committee of the Sapienza University of Rome and adhered to the tenets of the Declaration of Helsinki Principles. From this cohort, patients who were evaluated for at least 3 months after surgery and who met the inclusion and exclusion criteria were finally selected.

Inclusion criteria were as follows: (1) successfully repaired primary macula-off RRD by a single, uncomplicated SB surgical procedure, (2) follow-up examination at 1 month and every 3 months thereafter, and (3) patients who underwent cataract surgery during the postoperative follow-up period, at least 6 months of follow-up after cataract surgery. Exclusion criteria were as follows: (1) macular pathologic features, such as agerelated macular degeneration, vitreomacular traction syndrome, macular hole, ERM, and macular oedema, (2) a history of amblyopia in the affected eye, (3) diabetes and a history of retinal vascular occlusion in the affected eye, (4) a history of intraocular surgery other than uncomplicated cataract surgery, (5) visually significant cataract that was not treated with cataract surgery during the follow-up, (6) traumatic RRD, (7) RD associated with vitreous haemorrhage or giant retinal tears, and (8) patients with postoperative extensive defects of the photoreceptor inner/outer segment junction hyperreflective line as evaluated by SDOCT.

All SB surgeries were performed by two experienced retinal surgeons (MG and RM) at the Umberto I

University Hospital of Rome. After the positioning of an encircling silicone band, a circumferential silicone scleral explant was positioned to close the break(s). All eyes underwent external SRF drainage and cryotherapy application to the retinal break(s).

Before surgery, patients underwent a comprehensive ophthalmologic examination, including the measurement of Best-corrected visual acuity (BCVA), intraocular pressure, binocular indirect ophthalmoscopy, contact lens slit-lamp biomicroscopy, fundus photography, and OCT. Preoperative data collection included a complete medical and ophthalmic history, preoperative BCVA, characteristics of the RDs, lens status, and time from onset of the symptoms to surgery. The characteristics of the RDs and the presence of macular involvement were evaluated by binocular indirect ophthalmoscopy and slit-lamp biomicroscopy with the three-mirror Goldmann lens.

On each postoperative evaluation BCVA, complete ocular examination and OCT were performed. BCVA was measured using the standardized, 70-letter Early Treatment Diabetic Retinopathy Study chart (Chart ' $R$,' Precision Vision, La Salle, IL, USA) at $4 \mathrm{~m}$ distance. For statistical analysis, both preoperative and postoperative BCVA was analyzed on a logarithm of the minimal angle of resolution scale (logMAR). LogMAR values of 1.85 and 2.30 were assigned for counting fingers and hand motions vision at 2 feet, respectively.

SDOCT images were obtained, before and after surgery, with Spectralis OCT (Spectralis Family Acquisition Module, V 5.1.6.0; Heidelberg Engineering, Heidelberg, Germany), following a standardized protocol. Active eye-tracking (TruTrack, Heidelberg Engineering), and automatic follow-up scan (AutoRescan, Heidelberg Engineering) were used to enable point-topoint correspondence between consecutive followup scans.

Before surgery, a radial $30^{\circ}$, six lines scan protocol centred on the fovea were obtained for each eye with at least 16 frames averaged for each scan. The maximum height of the RD at the central fovea was defined as the distance between the outer surface of the detached neurosensory retina at the central fovea and the inner surface of the underlying retinal pigment epithelium. The height of the RD was measured using the digital caliper tool provided by the OCT software. The following preoperative retinal structural abnormalities were also evaluated: (1) cystoid changes in the inner nuclear layer, (2) cystoid or diffuse oedema in the outer nuclear layer, and (3) undulation of the photoreceptor layer.

After surgery, a raster horizontal $20^{\circ} \times 15^{\circ}, 19$ lines scan, with a reciprocal distance of $240 \mathrm{~mm}$, and a radial $20^{\circ}$, six lines scan protocol centred on the fovea were obtained for each eye. More than 25 scans were averaged for each scan. All scans were evaluated for the presence of persistent SRF. Persistent SRF was classified into two patterns as follows: (1) diffuse submacular fluid that extended beyond the edge of the 6-mm scan and (2) bleblike, localized pockets of fluid. Patients in whom the OCT appearance was compatible with bleb-like SRF were divided into three groups according to the pockets' localization with respect to the central 1-mm diameter 
area: (1) subfoveal bleb of fluid, (2) extrafoveal bleb(s) of fluid, and (3) mixed subfoveal and extrafoveal blebs of fluid. To evaluate the serial morphologic changes that characterize SRF bleb regression, we selected one SRF bleb from each eye then measured its maximum width and height at time of initial stage and during the course of follow-up until its disappearance. Two independent observers took each manual measurement, and the average of both values was used for data analysis.

\section{Statistical analysis}

Statistical analysis was performed with the SPSS for windows (V 17.0, SPSS, IBM Software Group, Chicago, IL, USA). Normal distribution of data was analyzed by the Kolmogorov-Smirnov test. Longitudinal data were compared using the paired $t$-test or the Wilcoxon test, as appropriate. Intergroup differences were evaluated using the unpaired $t$-test or the Mann-Whitney rank-sum test, as appropriate. Categorical variables were compared using Fisher's exact test. Bivariate relationships were evaluated by the Spearman's coefficient or the Pearson's analysis, as appropriate. In the correlation analysis, the following preoperative parameters has been evaluated as prognostic factors for persistence of SRF at 1 month after surgery and BCVA outcome: age, gender, symptoms duration, BCVA, lens status, RD extent, cystoid changes in the inner nuclear layer, cystoid or diffuse oedema in the outer nuclear layer, and undulation of the photoreceptor layer.
The predictive value of markers for SRF absorption was evaluated using receiver-operating characteristic (ROC) curve analysis.

Data are reported as mean values \pm SD. $P$-values of $<0.05$ were considered as statistically significant.

\section{Results}

Sixty-four eyes of 64 patients were included in this retrospective study. Forty-nine eyes completed the 6-month follow-up, 34 eyes completed the 9-month follow-up, and 27 eyes completed the 12-month follow-up to the time of results' evaluation. Patients' demographic and clinical characteristics at baseline are summarized in the Table 1.

In 10 patients, it was not possible to obtain adequate preoperative OCT images of the macula as the RD was highly elevated. For the same cause, in 28 patients, it was not possible to measure the height of detachment. Persistent SRF at 1 month after surgery was observed in 40 eyes $(62.5 \%)$. Six eyes $(9.4 \%)$ had diffuse SRF, 4 eyes (6.3\%) had subfoveal SRF bleb, 8 eyes $(12.5 \%)$ had extrafoveal blebs and 22 eyes (34.4\%) had mixed (subfoveal and extrafoveal) blebs. The cumulative survival curve of persistent SRF in all patients during follow-up was significantly different among SRF types $(P=0.03$; Figure 1).

Demographics and preoperative clinical characteristics were compared between eyes with and without persistent $\mathrm{SRF}$, no differences were found between groups in age, gender, symptoms duration, BCVA, lens status, RD

Table 1 Patients' demographics and comparisons of preoperative clinical characteristics between eyes with and without subretinal fluid at 1 month

\begin{tabular}{|c|c|c|c|c|}
\hline & $\begin{array}{l}\text { All sample } \\
\text { (64 eyes) }\end{array}$ & $\begin{array}{c}\text { SRF at } \\
1 \text { month (40 eyes) }\end{array}$ & $\begin{array}{l}\text { No SRF at } \\
1 \text { month ( } 24 \text { eyes) }\end{array}$ & P-value \\
\hline Age, years & $58.8 \pm 14.4$ & $57.2 \pm 15.5$ & $61.4 \pm 12.2$ & $0.3^{\mathrm{a}}$ \\
\hline Male/female & $39 / 25$ & $23 / 17$ & $16 / 8$ & $0.6^{\mathrm{b}}$ \\
\hline Symptoms duration, days & $7.3 \pm 5.7$ & $7.4 \pm 6.0$ & $7.0 \pm 5.4$ & $0.7^{c}$ \\
\hline Preoperative $\mathrm{BCVA}, \log \mathrm{MAR}$ & $1.4 \pm 0.8$ & $1.3 \pm 0.8$ & $1.6 \pm 0.7$ & $0.1^{\mathrm{c}}$ \\
\hline Phakic/pseudophakic & $43 / 21$ & $30 / 10$ & $13 / 11$ & $0.1^{\mathrm{b}}$ \\
\hline Retinal detachment extent, quadrants & $2.2 \pm 0.7$ & $2.2 \pm 0.7$ & $2.4 \pm 0.7$ & $0.3^{\mathrm{c}}$ \\
\hline $\begin{array}{l}\text { Maximum height of the retinal detachment, } \\
\text { microns (available data) }\end{array}$ & $787 \pm 321(36)$ & $747 \pm 317(20)$ & $837 \pm 329(16)$ & $0.3^{c}$ \\
\hline $\begin{array}{l}\text { Undulation of the photoreceptor layer, No. of eyes } \\
\text { (available data) }\end{array}$ & $43(54)$ & $25(33)$ & $18(21)$ & $0.5^{\mathrm{b}}$ \\
\hline $\begin{array}{l}\text { Cystoid changes in the inner nuclear layer, No. of eyes } \\
\text { (available data) }\end{array}$ & $21(54)$ & $10(32)$ & $11(22)$ & $0.3^{\mathrm{b}}$ \\
\hline $\begin{array}{l}\text { Cystoid or diffuse oedema in the outer nuclear layer, } \\
\text { No. of eyes (available data) }\end{array}$ & $49(54)$ & $30(33)$ & $19(21)$ & $1.0^{\mathrm{b}}$ \\
\hline
\end{tabular}

Abbreviations: BCVA, best-corrected visual acuity; logMAR, logarithm of the minimal angle of resolution; SRF, sub-retinal fluid.

Values are mean \pm SD unless otherwise indicated.

a Unpaired $t$-test with Levene's test for equality of variances.

${ }^{\mathrm{b}}$ Fisher's exact test.

${ }^{\mathrm{c}}$ Mann-Whitney U-test. 


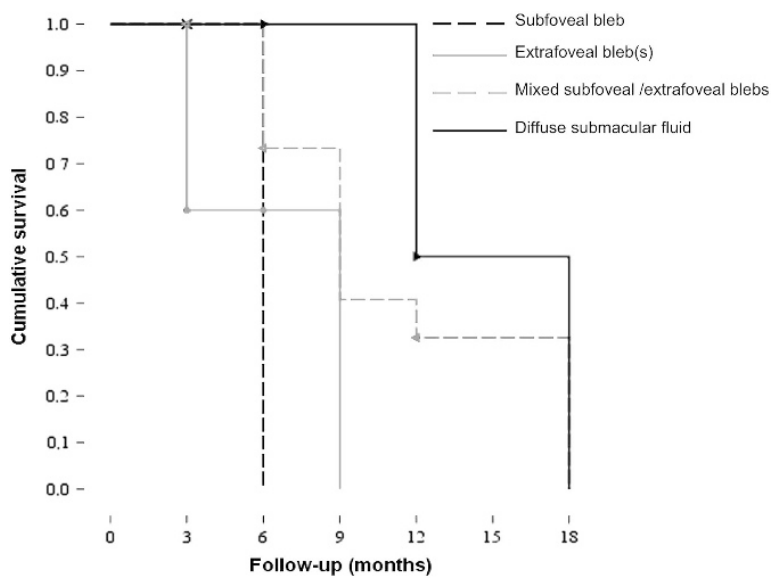

Figure 1 Cumulative survival curve of persistent subretinal fluid after scleral buckling surgery for macula-off rhegmatogenous retinal detachment. Arrowhead marks indicate censored (incomplete optical coherence tomography follow-up) cases.

extent, maximum height of the RD, and any of the preoperative retinal structural abnormalities as evaluated by SDOCT $(P>0.05$; Table 1$)$.

Of six eyes with confluent SRF at 1 month, SRF pattern turned from diffuse to bleb type in five cases during follow-up. In the remaining case it was no longer detectable at month 6 after surgery. Of 40 patients with persistent SRF at 1 month, we were able to verify the complete absorption of SRF with SDOCT during the follow-up period in 29 cases. SRF blebs were first recognizable at $1.7 \pm 2.2$ months postoperatively. SRF blebs were completely absorbed within 1 year after surgery in 26 eyes (90\%) and within 18 months postoperatively in the remaining 3 eyes $(10 \%)$. The average time for complete SRF bleb absorption was $7.8 \pm 4.4$ months postoperatively.

Mean SRF bleb height was $86 \pm 30 \mu \mathrm{m}$ at 1 month, $104 \pm 42 \mu \mathrm{m}$ at 3 months, $94 \pm 39 \mu \mathrm{m}$ at 6 months, $79 \pm 33 \mu \mathrm{m}$ at 9 months, and $100 \pm 55 \mu \mathrm{m}$ at 12 months. Mean SRF bleb width was $646 \pm 329 \mu \mathrm{m}$ at 1 month, $710 \pm 495 \mu \mathrm{m}$ at 3 months, $745 \pm 483 \mu \mathrm{m}$ at 6 months, $555 \pm 246 \mu \mathrm{m}$ at 9 months, and $538 \pm 478 \mu \mathrm{m}$ at 12 months. Compared with the initial stage, bleb width decreased significantly at all time points during the 12-month follow-up period $(P<0.05)$, whereas significant bleb height decrease occurred from postoperative sixth month only $(P<0.05)$. At 3 months, the percentage change of bleb height $v s$ width was significantly different $(P=0.03$; Figure 2). A representative case showing the natural course of residual SRF blebs after RRD surgery is given in Figure 3.

Using the ROC curve analysis we found that the cutoff value of the bleb width-to-height ratio level for predicting bleb absorption at 6 months was 7 , with $89 \%$ sensitivity and $83 \%$ specificity. The area under the ROC curve for

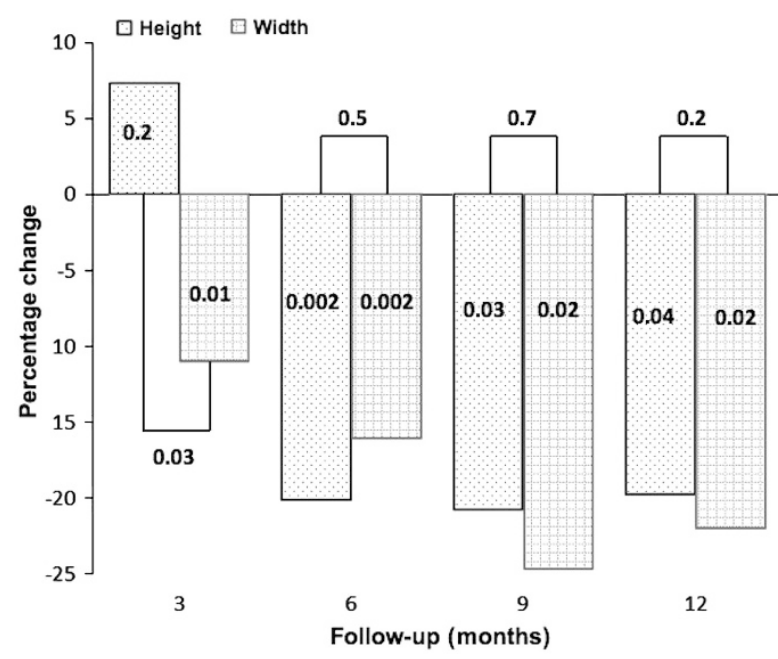

Figure 2 Serial SDOCT analysis of residual subretinal fluid blebs after scleral buckling surgery for macula-off rhegmatogenous retinal detachment. Bar-graph showing the percentage variation of subretinal fluid bleb height and width. $P$-values inside the bars result from the paired $t$-test, whereas those outside the bars result from the unpaired $t$-test.

bleb width-to-height ratio was $0.944(P=0.0001,95 \%$ CI: 0.87-1).

Overall, compared with baseline, BCVA had improved significantly at all time points $(P<0.0001)$. After surgery, BCVA was $0.35 \pm 0.28 \log \mathrm{MAR}$ at 1 month, $0.23 \pm 0.22$ $\log \mathrm{MAR}$ at 3 months, $0.20 \pm 0.22 \log \mathrm{MAR}$ at 6 months, $0.16 \pm 0.22 \log \mathrm{MAR}$ at 9 months, and $0.13 \pm 0.17 \log \mathrm{MAR}$ at 12 months. BCVA outcome was found to be correlated with the maximum height of the RD before surgery ( $r=0.46, P=0.006$ at 1 month; $r=0.59, P<0.0001$ at 3 months; $r=0.64, P<0.0001$ at 6 months; $r=0.58$, $P=0.005$ at 9 months; $r=0.58, P=0.007$ at 12 months) and with RD extent ( $r=0.32, P=0.01$ at 3 months; $r=0.32$, $P=0.02$ at 6 months; $r=0.45, P=0.005$ at 9 months; $r=0.42, P=0.02$ at 12 months). We found little evidence of any association between BCVA outcome and each of age, gender, symptoms duration, preoperative BCVA, lens status, and the preoperative retinal structural abnormalities as evaluated by SDOCT $(P>0.05)$.

Subgroup analysis of cases with and without subfoveal fluid revealed that the greatest improvement in BCVA was seen within the first 3 months $(P<0.001)$, although visual acuity continued to improve significantly until 12 months in both groups $(P<0.05$; Figure 4$)$. There was no difference between groups in BCVA outcomes during the follow-up period $(P>0.05)$. In those eyes with persistent subfoveal fluid, the disappearance of SRF was associated with an improvement in BCVA, mean BCVA before and 3-month after SRF absorption was $0.26 \pm 0.27$ $\log$ MAR and $0.14 \pm 0.18 \log M A R$, respectively $(P=0.003)$. 


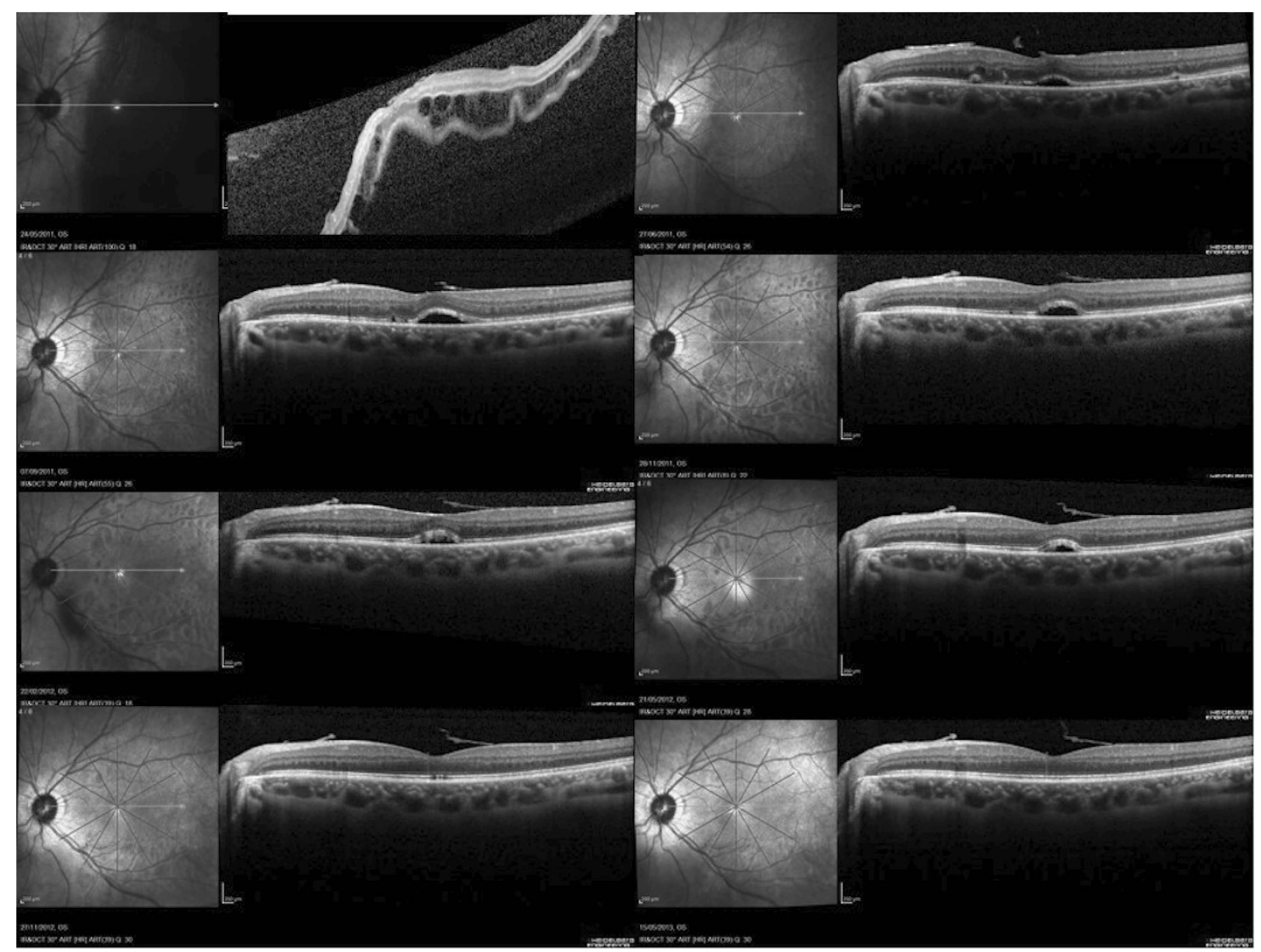

Figure 3 Serial fundus infrared reflectance (IR) and spectral-domain optical coherence (SDOCT) tomography images showing the natural course of residual SRF blebs after scleral buckling surgery for macula-off rhegmatogenous retinal detachment (RRD). (Top left) Preoperative IR and SDOCT images of a 37-year-old patient with macula-off RRD. Undulation of the photoreceptor layer and severe cystoid changes in the outer nuclear layer were present. Visual acuity was counting fingers. (Top right) At 1 month postoperatively, SRF blebs were observed. Visual acuity was $0.32 \log$ MAR. (Second row left) Three months after surgery, visual acuity was 0.32 logMAR. (Second row right) At 6 months, visual acuity was $0.24 \log$ MAR. (Third row left) At 9 months, visual acuity was $0.18 \log$ MAR. Note the progressive elongation of the photoreceptors' outer segments. (Third row right) One year after surgery, visual acuity was 0.18 logMAR. Note the bleb width decrease. (Bottom left) At 18 months, visual acuity was 0.08 logMAR. The SRF bleb finally regressed leaving focal defects in the photoreceptors' inner segment/outer segment (IS/OS) junction line. (Bottom right) Two years after surgery, visual acuity was $0.08 \log$ MAR. Note the apparent complete recovery of the IS/OS junction band.

We found no association between BCVA outcomes and subfoveal bleb height or width $(P>0.05)$.

\section{Discussion}

Residual 'pockets' of SRF are known to occur after SB surgery for RRD. In this study, we found that subfoveal located blebs may slow visual recovery, although final outcome was not influenced. We further demonstrated that a bleb width-to-height ratio $>7$ indicates a higher risk of SRF to persist beyond 6 months after surgery.

The incidence of persistent SRF after SB for primary RRD varies widely, ranging from 9 to $94 \%$, depending on the reported series. ${ }^{2-13}$ This variability among studies is probably related to differences in study design, baseline characteristics of patients and operating procedures. Our retrospective series demonstrated that $62.5 \%$ of eyes with macula-off RRD had persistent SRF on OCT at 1 month after surgery and this finding is consistent with most previous reports in this setting. Ricker et al ${ }^{13}$ found persistent subfoveal fluid on OCT in up to $94 \%$ of patients with macula-off RRD at 1-month follow-up. This exceptionally high incidence may be ascribed to the operating procedure. Indeed, $76 \%$ of patients in Ricker's series underwent intraoperative SF6 gas injection that could have induced subfoveal fluid displacement.

Younger age, phakia, and longer standing inferior detachment all have been suggested as possible preoperative risk factors for persistent SRF after SB surgery without, however, conclusive evidence. ${ }^{14} \mathrm{We}$ found no association between persistence of SRF and these aforementioned factors. Clinical practice 


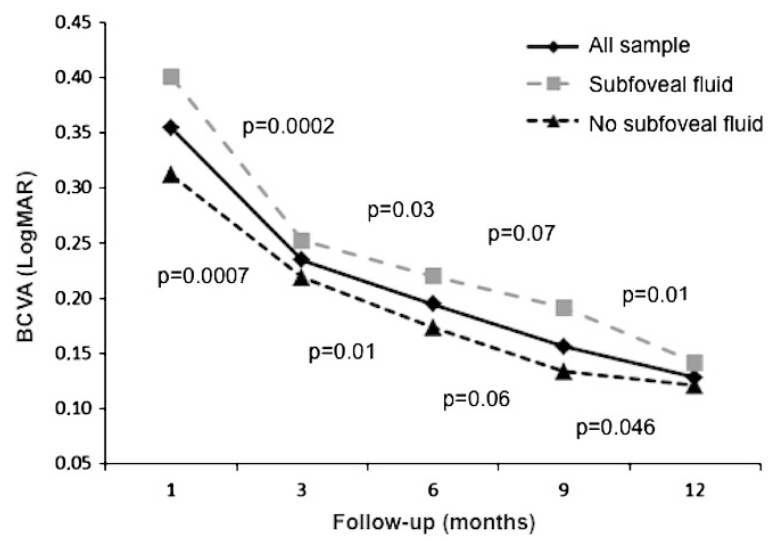

Figure 4 Best-corrected visual acuity (BCVA) outcomes over time after scleral buckling surgery for macula-off rhegmatogenous retinal detachment. Upper and lower $P$-values result from comparisons between consecutive follow-up steps in eyes with and without subfoveal fluid, respectively (Wilcoxon paired test).

demonstrates that longstanding detachments with viscous SRF tend to show delayed fluid absorption. However, this relationship is difficult to prove, as data on the duration of RD are usually recorded retrospectively, relying on patient recollection as to the start of the symptoms, which may be markedly inaccurate.

In our results, the average time for complete SRF bleb absorption was $7.8 \pm 4.4$ months postoperatively. Compared with subfoveal blebs, diffuse SRF and mixed (subfoveal and extrafoveal) blebs tended to persist longer over time. It is not surprising that the rate of SRF absorption is related to the amount of persistent fluid.

Serial OCT analysis of residual SRF blebs showed that, compared with the initial stage, bleb width decreased significantly at all time points during the 12-month follow-up period, whereas significant bleb height decrease occurred from the sixth month only. These results are consistent with those reported in a recent retrospective study evaluating the temporal morphological changes of multiple SRF blebs. ${ }^{15}$ During the natural course, the authors found a progressive bleb width decrease and height increase. This may result from the active reattachment of retinal pigment epithelium and photoreceptors at the margin of residual SRF bleb. ${ }^{16}$ Experimental studies on animal models of RD showed a number of degenerative changes of the RPE, loss of apical villi, disappearance of phagosome from the cytoplasm, and misplacement of melanin granules and mitochondria. These morphologic changes imply that metabolic changes also occur in these cells in response to their loss of contact with photoreceptor outer segments. ${ }^{17,18}$ Furthermore, RPE proliferation with loss of surface polarity and Muller cell proliferation has also been described in the subretinal space. ${ }^{17,19}$ All these changes persisted, to some extent, in the reattached retina and have been described as having a 'patchwork' appearance. ${ }^{20}$ This refers to the fact that the anatomical recovery across the RPE is not uniform, and some areas may lag behind others in forming a close photoreceptor-RPE apposition. Therefore, bleb-like pockets of persistent SRF may represent areas of residual RPE dysfunction (eg, decreased phagocytotic activity and/or active transport of ions). A further hypothesis, that does not exclude the previous, is related to the SRF composition. Indeed, biochemical and histological analysis have shown that large molecules such as proteins and GAG, as well as cells and cellular debris are concentrated particularly in longstanding SRF. ${ }^{14,21}$ These compounds cannot be removed through ion or water channels, resulting in an imbalance between the decreased capability of and the increased need for phagocytosis by RPE cells.

We observed a negative association between BCVA outcome and both the preoperative height and extent of $\mathrm{RRD}$. These findings are consistent with those of experimental studies on animal model that showed more progressive outer retinal damage with greater height of the retinal detachment. ${ }^{1,17,22}$ Accordingly, clinical studies, evaluating the morphology of the detached macula in primary RRD by SDOCT, showed that higher retinal detachment at the fovea was correlated with dropout of foveal photoreceptor IS/OS layer and worse postoperative VA. ${ }^{23,24}$

Although we did not find any significant difference in VA outcome between eyes with persistent subfoveal fluid and those without subfoveal fluid, subgroup analysis revealed that in those eyes with residual subfoveal fluid, the disappearance of fluid was associated with an improvement in visual acuity. Our results, along with those of Benson et al ${ }^{8}$ and Seo et al, ${ }^{10}$ suggest that there is an association of delayed visual recovery in patients with macula-off RRD and residual SRF; however, the final visual outcome does not seem to be affected. In addition, we found no association between height or width of subfoveal SRF and VA outcome. Mean height of subfoveal SRF in our series was relatively stable at $\sim 90 \mu \mathrm{m}$ until SRF disappeared. Similar to our results, Seo et a $1^{10}$ observed that persistent SRF of $\sim 100 \mu$ m height within 12 months may slow visual recovery but does not cause VA loss after SB surgery for macula-off RRD. However, BCVA, which was used in our study as well as in previous reports, might not be enough to evaluate the detailed visual function. Indeed, Okamoto et al, ${ }^{25}$ in a recent study evaluating metamorphopsia after RRD surgery, found that metamorphopsia was more severe in eyes with persistent submacular fluid. Further studies using other measurement of visual function such as microperimetry and contrast sensitivity can better evaluate the actual clinical implications of persistent SRF in this setting. 
The limitations of the present study are its retrospective design and the small sample size.

In this retrospective series, our results showed that $62.5 \%$ of eyes with macula-off RRD had persistent SRF on OCT at 1 month after SB surgery. Visual improvement may occur with late resolution of residual subfoveal fluid.

Serial morphological analysis of residual subfoveal bleb showed no relation between VA outcomes and the bleb size. We further demonstrated that a bleb width-to-height ratio $>7$ indicates a higher risk of SRF to persist beyond 6 months after surgery. This clinical information may have valuable implications for the patient's prognosis and may allow clinicians to better address patients' expectations of visual recovery after surgery.

\section{Summary}

\section{What was known before}

- Persistent subretinal fluid has been reported after retinal detachment surgery, however, it remains unclear whether the visual acuity outcome is affected.

- Only one SDOCT study analysed the serial changes of SRF bleb size during the natural course. A progressive bleb width decrease and height increase was observed.

\section{What this study adds}

- There was no difference in visual acuity outcomes between cases with persistent subfoval fluid and those without fluid. However, the disappearance of residual subfoveal fluid was associated with an improvement of visual acuity.

- A bleb width-to-height ratio $>7$ indicates a higher risk of SRF to persist beyond 6 months after surgery.

- Our study showed that compared with the initial stage, bleb width decreased significantly at all time points during the 12-month follow-up period, whereas significant bleb height decrease occurred from postoperative sixth month only. This suggests that SRF absorption is mediated by the photoreceptor-EPR complex active metabolism at the margin of residual SRF bleb.

\section{Conflict of interest}

The authors declare no conflict of interest.

\section{References}

1 Machemer R. Experimental retinal detachment in the owl monkey. II. Histology of retina and pigment epithelium. Am J Ophthalmol 1983; 95: 182-188.

2 Kaga T, Fonseca RA, Dantas MA, Yannuzzi LA, Spaide RF. Optical coherence tomography of bleb-like subretinal lesions after retinal reattachment surgery. Am J Ophthalmol 2001; 132: 120-121.

3 Hagimura N, Iida T, Suto K, Kishi S. Persistent foveal retinal detachment after successful rhegmatogenous retinal detachment surgery. Am J Ophthalmol 2002; 133: 516-520.

4 Wolfensberger TJ, Gonvers M. Optical coherence tomography in the evaluation of incomplete visual acuity recovery after macula-off retinal detachments. Graefes Arch Clin Exp Ophthalmol 2002; 240: 85-89.

5 Wolfensberger TJ. Foveal reattachment after macular-off retinal detachment occurs faster after vitrectomy than after buckle surgery. Ophthalmology 2004; 111: 1340-1343.

6 Baba T, Hirose A, Moriyama M, Mochizuki M. Tomographic image and visual recovery of acute macula-off rhegmatogenous retinal detachment. Graefes Arch Clin Exp Ophthalmol 2004; 242: 576-581.

7 Gibran SK, Alwitry A, Cleary PE. Foveal detachment after successful retinal reattachment for macula on rhegmatogeneous retinal detachment: an optical coherence tomography evaluation. Eye 2006; 20: 1284-1287.

8 Benson SE, Schlottmann PG, Bunce C, Xing W, Charteris DG. Optical coherence tomography analysis of the macula after scleral buckle surgery for retinal detachment. Ophthalmology 2007; 114: 108-112.

9 Benson SE, Schlottmann PG, Bunce C, Xing W, Charteris DG. Optical coherence tomography analysis of the macula after vitrectomy surgery for retinal detachments. Ophthalmology 2006; 113: 1179-1183.

10 Seo JH, Woo SJ, Park KH, Yu YS, Chung H. Influence of persistent submacular fluid on visual outcome after successful scleral buckle surgery for macula-off retinal detachment. Am J Ophthalmol 2008; 145: 915-922.

11 Kang SW, Kim JH, Shin WJ, Kim JI. Subretinal fluid bleb after successful scleral buckling and cryotherapy for retinal detachment. Am J Ophthalmol 2008; 146: 205-210.

12 Kim YK, Woo SJ, Kim KE, ParK KH. Subretinal fluid resorption. Ophthalmology 2010; 117: 1655, 1655.e1-e4.

13 Ricker LJ, Noordzij LJ, Goezinne F, Cals DW, Berendschot TT, Liem AT et al. Persistent subfoveal fluid and increased preoperative foveal thickness impair visual outcome after macula-off retinal detachment repair. Retina 2011; 31: 1505-1512.

14 Veckeneer M, Derycke L, Lindstedt EW, van Meurs J, Cornelissen M, Bracke M et al. Persistent subretinal fluid after surgery for rhegmatogenous retinal detachment: hypothesis and review. Graefes Arch Clin Exp Ophthalmol 2012; 250: 795-802.

15 Kim YK, Ahn J, Woo SJ, Hwang DJ, Park KH. Multiple subretinal fluid blebs after successful retinal detachment surgery: incidence, risk factors, and presumed pathophysiology. Am J Ophthalmol 2014; 157: 834-841.

16 Negi A, Marmor MF. The resorption of subretinal fluid after diffuse damage to the retinal pigment epithelium. Invest Ophthalmol Vis Sci 1983; 24: 1475-1479.

17 Anderson DH, Stern WH, Fisher SK, Erickson PA, Borgula GA. Retinal detachment in the cat: the pigment epithelial-photoreceptor interface. Invest Ophthalmol Vis Sci 1983; 24: 906-926.

18 Immel J, Negi A, Marmor MF. Acute changes in RPE apical morphology after retinal detachment in rabbit. A SEM study. Invest Ophthalmol Vis Sci 1986; 27: 1770-1776.

19 Sethi CS, Lewis GP, Fisher SK, Leitner WP, Mann DL, Luthert PJ et al. Glial remodeling and neural plasticity in human retinal detachment with proliferative vitreoretinopathy. Invest Ophthalmol Vis Sci 2005; 46: 329-342.

20 Anderson DH, Guérin CJ, Erickson PA, Stern WH, Fisher SK. Morphological recovery in the reattached retina. Invest Ophthalmol Vis Sci 1986; 27: 168-183. 
21 Quintyn JC, Brasseur G. Subretinal fluid in primary rhegmatogenous retinal detachment: physiopathology and composition. Surv Ophthalmol 2004; 49: 96-108.

22 Erickson P, Fisher S, Anderson D, Stern WH, Borgula GA. Retinal detachment in the cat: the outer nuclear and the outer plexiform layers. Invest Ophthalmol Vis Sci 1983; 24: 927-942.

23 Lecleire-Collet A, Muraine M, Menard JF, Brasseur G. Predictive visual outcome after macula-off retinal detachment surgery using optical coherence tomography. Retina 2005; 25: 44-53.

24 Nakanishi H, Hangai M, Unoki N, Sakamoto A, Tsujikawa A, Kita $\mathrm{M}$ et al. Spectral-domain optical coherence tomography imaging of the detached macula in rhegmatogenous retinal detachment. Retina 2009; 29: 232-242.

25 Okamoto F, Sugiura Y, Okamoto Y, Hiraoka T, Oshika T. Metamorphopsia and optical coherence tomography findings after rhegmatogenous retinal detachment surgery. Am J Ophthalmol 2014; 157: 214-220. 